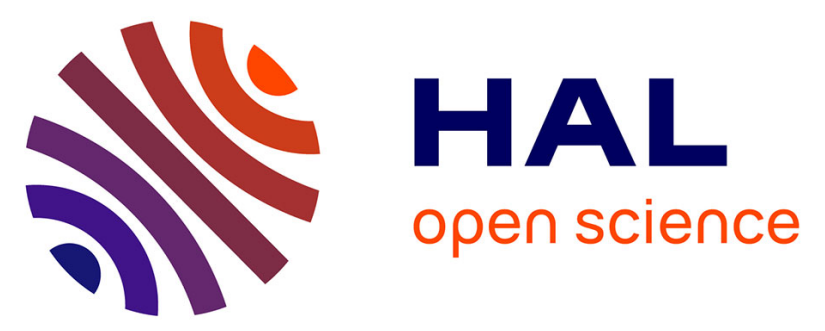

\title{
Early toxicity of a phase II trial of combined salvage radiotherapy and hormone therapy in oligometastatic pelvic node relapses of prostate cancer (OLIGOPELVIS GETUG P07)
}

Loig Vaugier, Clément Palpacuer, Emmanuel Rio, Aurore Goineau, David Pasquier, Xavier Buthaud, Guy de Laroche, Véronique Beckendorf, Paul Sargos, Gilles Créhange, et al.

\section{- To cite this version:}

Loig Vaugier, Clément Palpacuer, Emmanuel Rio, Aurore Goineau, David Pasquier, et al.. Early toxicity of a phase II trial of combined salvage radiotherapy and hormone therapy in oligometastatic pelvic node relapses of prostate cancer (OLIGOPELVIS GETUG P07). International Journal of Radiation Oncology, Biology, Physics, 2018, 103 (5), pp.1061-1067. 10.1016/j.ijrobp.2018.12.020 . inserm-01980788

\section{HAL Id: inserm-01980788 https://www.hal.inserm.fr/inserm-01980788}

Submitted on 14 Jan 2019

HAL is a multi-disciplinary open access archive for the deposit and dissemination of scientific research documents, whether they are published or not. The documents may come from teaching and research institutions in France or abroad, or from public or private research centers.
L'archive ouverte pluridisciplinaire HAL, est destinée au dépôt et à la diffusion de documents scientifiques de niveau recherche, publiés ou non, émanant des établissements d'enseignement et de recherche français ou étrangers, des laboratoires publics ou privés. 


\section{Accepted Manuscript}

Early toxicity of a phase II trial of combined salvage radiotherapy and hormone therapy in oligometastatic pelvic node relapses of prostate cancer (OLIGOPELVIS GETUG P07)

Loig Vaugier, Clément Palpacuer, Emmanuel Rio, Aurore Goineau, David Pasquier,

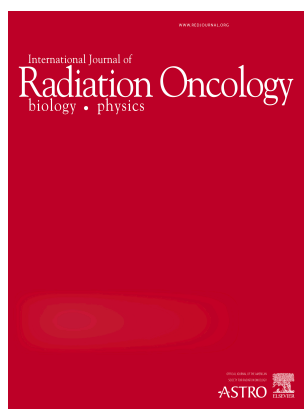
Xavier Buthaud, Guy De Laroche, Véronique Beckendorf, Paul Sargos, Gilles Créhange, Pascal Pommier, Geneviève Loos, Ali Hasbini, Igor Latorzeff, Marlon Silva, Fabrice Denis, Jean-Léon Lagrange, Loic Campion, Stéphane Supiot

PII: S0360-3016(18)34186-5

DOI: https://doi.org/10.1016/j.jijrobp.2018.12.020

Reference: ROB 25452

To appear in: International Journal of Radiation Oncology • Biology • Physics

Received Date: 22 June 2018

Revised Date: 4 December 2018

Accepted Date: 9 December 2018

Please cite this article as: Vaugier L, Palpacuer C, Rio E, Goineau A, Pasquier D, Buthaud X, De Laroche G, Beckendorf V, Sargos P, Créhange G, Pommier P, Loos G, Hasbini A, Latorzeff I, Silva M, Denis F, Lagrange J-L, Campion L, Supiot S, Early toxicity of a phase II trial of combined salvage radiotherapy and hormone therapy in oligometastatic pelvic node relapses of prostate cancer (OLIGOPELVIS GETUG P07), International Journal of Radiation Oncology • Biology • Physics (2019), doi: https://doi.org/10.1016/j.ijrobp.2018.12.020.

This is a PDF file of an unedited manuscript that has been accepted for publication. As a service to our customers we are providing this early version of the manuscript. The manuscript will undergo copyediting, typesetting, and review of the resulting proof before it is published in its final form. Please note that during the production process errors may be discovered which could affect the content, and all legal disclaimers that apply to the journal pertain. 


\section{Early toxicity of a phase II trial of combined salvage radiotherapy and}

\section{hormone therapy in oligometastatic pelvic node relapses of prostate \\ cancer (OLIGOPELVIS GETUG P07)}

\section{Short title : Early toxicity of the OLIGOPELVIS GETUG P07 trial}

Loig Vaugier ${ }^{1}$, Clément Palpacuer ${ }^{2}$, Emmanuel Rio $^{1}$, Aurore Goineau ${ }^{3}$, David Pasquier ${ }^{4,5}$ Xavier Buthaud $^{6}$, Guy De Laroche ${ }^{7}$, Véronique Beckendorf ${ }^{8}$, Paul Sargos $^{9}$, Gilles Créhange ${ }^{10}$, Pascal Pommier ${ }^{11}$, Geneviève Loos ${ }^{12}$, Ali Hasbini ${ }^{13}$ Igor Latorzeff $^{14}$, Marlon Silva ${ }^{15}$, Fabrice Denis ${ }^{16}$, Jean-Léon Lagrange ${ }^{17}$, Loic Campion ${ }^{2,18}$ and Stéphane Supiot* $*^{1,18}$

${ }^{1}$ Department of Radiation Oncology, Institut de Cancérologie de l'Ouest, Boulevard J. Monod, F44800 Nantes, St-Herblain France

${ }^{2}$ Department of Biostatistics, Institut de Cancérologie de I Ouest, Boulevard J. Monod, F-44800 Nantes, St-Herblain France

${ }^{3}$ Department of Radiation Oncology, Institut de Cancérologie de l'Ouest, Angers, France

${ }^{4}$ Academic Radiation Oncology Department, Centre Oscar Lambret, Lille, France

${ }^{5}$ Centre de Recherche en Informatique, Signal et Automatique de Lille, CRIStAL UMR CNRS

9189, Université de Lille, France

${ }^{6}$ Department of Radiation Oncology, Centre Catherine de Sienne, Nantes, France

${ }^{7}$ Department of Radiation Oncology, Institut de Cancérologie de la Loire, St Priest en Jarez, France

${ }^{8}$ Department of Radiation Oncology, Centre Alexis Vautrin, Vandoeuvre-lès-Nancy, France

${ }^{9}$ Department of Radiation Oncology, Institut Bergonié, Bordeaux, France

${ }^{10}$ Department of Radiation Oncology, Georges-Francois Leclerc Cancer Center, Dijon, France

${ }^{11}$ Department of Radiation Oncology, Centre Léon Bérard, Lyon, France 
${ }^{12}$ Department of Radiation Oncology, Centre Jean Perrin, Clermont-Ferrand, France

${ }^{13}$ Department of Radiation Oncology, Clinique Pasteur, Brest, France

${ }^{14}$ Department of Radiation Oncology, Oncorad Clinique Pasteur, Toulouse, France

${ }^{15}$ Department of Radiation Oncology, Centre Francois Baclesse, Caen, France

${ }^{16}$ Department of Radiation Oncology, Centre Jean Bernard, Le Mans, France

${ }^{17}$ Department of Radiation Oncology, Hopital Henri Mondor, Créteil, France

${ }^{18}$ Centre de Recherche en Cancérologie Nantes-Angers (CRCNA), UMR 1232 Inserm - 6299

CNRS, Institut de Recherche en Santé de l'Université de Nantes, 8 quai Moncousu, BP 70721, 44007 Nantes Cedex 1, France

*corresponding author : stephane.supiot@ico-unicancer.fr

Department of Radiation Oncology, Institut de Cancérologie de l`Ouest, Boulevard J. Monod, F44800 Nantes, St-Herblain France

\section{Authors responsible for statistical analyses :}

Clément Palpacuer : clement.palpacuer@ico-unicancer.fr

Loic Campion : loic.campion@ico-unicancer.fr

Acknowledgements : This study was funded by Astellas. 
Early toxicity of a phase II trial of combined salvage radiotherapy and hormone therapy in oligometastatic pelvic node relapses of prostate cancer (BLINDED) 


\section{Abstract}

\section{Purpose :}

Limited pelvic nodal relapse of prostatic cancer is a paramount challenge for locoregional salvage treatments. Salvage whole pelvis radiotherapy as considered in the BLINDED trial, is an attractive option but with concerns about its toxicity. This article describes early toxicity with the technique.

\section{Methods and Materials :}

BLINDED was a prospective multi-center phase II trial investigating high-dose salvage pelvic irradiation with an additional dose to the fluorocholine-based positron-emission-tomography (FCHPET)-positive pelvic lymph nodes (PLN), combined with six-month androgen blockade. The prescribed dose was 54 Gy in 1.8 Gy fractions with up to 66 Gy in 2.2 Gy fractions to the pathological PLN. Early toxicity was defined until one year after radiotherapy. Patients quality of life was assessed using the EORTC questionnaires (QLQ-C30 and QLQ-PR25).

\section{Results :}

Seventy-four patients were recruited in fifteen French radiation oncology departments between August 2014 and July 2016. Seven were excluded before treatment because of violation of the inclusion criteria. The intention-to-treat analysis therefore included sixty-seven patients. Half of them had received prior prostatic irradiation. Median age was $67.7 \pm 6.5$ years. Grade 2 acute urinary toxicity was observed in 9/67 patients (13.4\%) and grade 2 one-year toxicity in 4/67 patients (6\%). Three patients (4.4\%) had grade 3 urinary toxicity. Grade 2 acute digestive toxicity was observed in $10 / 67$ patients $(14.9 \%)$ and grade 2 one-year toxicity in $4 / 67$ patients $(6 \%)$. Patients with prior prostate bed irradiation did not exhibit increased urinary or digestive toxicity. EORTC questionnaire scores at one year did not worsen significantly.

\section{Conclusions :}

The acute and one-year toxicity of the BLINDED protocol was satisfactory, even in patients with a 
past history of prostatic irradiation.

Keywords : pelvis salvage irradiation; urinary toxicity; bowel toxicity; prostate cancer; IMRT; IGRT; Fluorocholine PET; PSMA PET; pelvic reirradiation 


\section{Introduction}

The development of new imaging techniques based on prostate-cancer specific markers such as fluorocholine positron-emission tomography (FCH-PET), has made identification of limited metastatic relapses of prostatic cancer feasible [1]-[3]. Among the various oligometastatic scenarios - a limited number of metastases $(\leq 5$ bone and/or lymph node metastases, with no visceral involvement) after previous prostate treatment - a pelvic lymph node (PLN) relapse is a paramount challenge, as an apparent turning point between still-controllable locoregional disease that can be managed without androgen blockade (through with salvage therapeutics) and diffuse disease for which androgen blockade would be the most appropriate treatment [4]-[5].

Salvage whole pelvis radiotherapy (WPRT) with an additional boost to any FCH-PET-positive PLN is an attractive option, but the best current evidence available is derived from retrospective studies on heterogeneous populations with heterogeneous treatment plans, though urinary and digestive toxicity was apparently acceptable [6]-[8]. Last but not least, despite prior prostatic bed radiotherapy as a first-line salvage treatment after radical prostatectomy or prostate-exclusive radiotherapy, a number of patients fulfilled the criteria of pelvic oligometastatic disease, and thus would potentially benefit from salvage pelvic reirradiation [9]. The question of the toxicity in this circumstance is even more pertinent.

The main objective of the multi-center phase II BLINDED trial (NCT BLINDED) [10] was to assess the efficacy of high-dose salvage WPRT in a prospective manner in a well-defined population. Prior prostatic irradiation was allowed. Here we present the early toxicity of this treatment, ie until one year after radiotherapy. 


\section{Materials and Methods}

The BLINDED trial design has already been published [10]. The trial population was divided into four groups, each with a different treatment plan (see fig.1 for planning doses):

- Group A: prior radical prostatectomy and no prior prostate bed radiation, with fewer than five FCH-PET-positive PLN;

- Group B: as group A, but with also a FCH-PET-positive signal in the prostate bed, suggesting local relapse;

- Group C: with both previous radical prostatectomy and salvage prostate bed radiotherapy, thus entering a second round of salvage therapy in the BLINDED trial;

- Group D : with prior conservative prostate treatment (external-body radiation or brachytherapy).

Image-guided intensity-modulated radiation (IG-IMRT) was required to deliver 54 Gy in 1.8 Gy fractions, with up to 66 Gy in 2.2 Gy fractions to the pathological PLN with simultaneous integrated boost (SIB). Patients who had not received prior irradiation, received 66 Gy in 2 Gy fractions to the prostatic bed, with up to $72 \mathrm{~Gy}$ in 2 Gy fractions in the case of prostatic bed local relapse. Androgen blockade was achieved by LH-RH agonist or antagonist injections during six months, ideally administered on the first day of radiotherapy, or within the three months prior to the first day of radiotherapy [10].

Acute toxicity was defined as events occurring between the first week of radiotherapy and one month after the end of radiotherapy (M1). Later events were documented from M1 until one year after radiotherapy in the present study. If a patient presented the same toxic event several times, only the higher grade event was analyzed. All toxicities were graded according to the CTCAE v4.0 classification. 
Patient quality of life was evaluated at inclusion (baseline) and at M6 with the QLQ-C30 v3 and the prostate cancer module QLQ-PR25 questionnaires of the EORTC [11].

Sixty-three $(+10 \%)$ evaluable patients were required to achieve adequate statistical power [10]. The primary outcome (not reported here) was biochemical relapse-free survival at two years.

Data from all evaluable patients was analyzed. Baseline and six-month quality of life scores were compared using a Wilcoxon signed test for matched pairs. A Benjamini-Hochberg procedure was applied to control for false discovery rate. For all analyses, a p-value of less than 0.05 was considered as statistically significant. All reported p-values are two sided. Quality of life differences were considered as clinically relevant when greater than 10 [11].

\section{Results}

Seventy-five patients in 15 French oncology centers were assessed for eligibility from August 2014 until July 2016. Sixty-seven patients (median age, $67.7 \pm 6.5$ years) were analyzed in intention-totreat (fig. 2). Patient characteristics and staging at diagnosis are summarized in table 1. Sixty-one patients (91\%) were initially treated by radical prostatectomy (groups A, B and C). Twenty-nine of the sixty-seven patients $(43.3 \%)$ received first-line salvage prostate bed radiation (group C). Only a minority of the patients $(9 \%, 6 / 67)$ had been previously treated conservatively (Group D): three were treated with external-beam radiotherapy at a mean dose of 74 Gy (70-76 Gy) and three had received prostate brachytherapy. A huge majority (83.5\%) had one or two positive pelvic lymph nodes at relapse. Four patients in group B, with no prior radiotherapy, had a local relapse in the prostate bed.

Acute genitourinary toxicity was dominated by grade 1 urinary urgency (33/67 patients, 49.2\%) (fig. 
3). The frequency of genito-urinary events at one year globally decreased in comparison to M1

(32.8\% (22/67) grade 1 urinary urgency; $3 \%(2 / 67)$ grade $2 ; 25.3 \%(17 / 67)$ grade 1 urinary incontinence). Three patients (4.4\%) reported grade 3 urinary incontinence and one of them grade 3 hematuria. No urinary incontinence was then reported at one year but grade 2 hematuria and grade 2 urinary urgency ; one group B patient developped grade 3 urinary incontinence with grade 3 hematuria at one year, leading to the discovery of a bladder papillary carcinoma (pTa); one group C patient reported isolated grade 3 urinary incontinence at one year without earlier symptoms.

Around $67 \%$ of the patients (45/67) were affected by acute moderate diarrhea: $55.2 \%(37 / 67)$ grade 1 and $11.9 \%(8 / 67)$ grade 2 . Around $34 \%$ of the patients (23/67) reported moderate grade 1 abdominal pain, constipation, bloating or flatulence. At one year : around $30 \%(20 / 67)$ reported grade 1 digestive inconvenience. There was only one grade $2(1.5 \%)$ diarrhea and one grade 2 $(1.5 \%)$ anal without abdominal upset. Two patients (3\%) suffered from grade 2 rectal bleeding.

Pooling the patients who had not previously undergone radiotherapy (groups A and B) versus the others (groups C and D), there were no notable differences regarding the acute or later toxicity (fig. 3 and supplementary for details).

There were no cardiovascular events, but a moderate worsening of hypertension.

Regarding the quality of life evaluation : the completion rates for the QLQ-C30 and QLQ-PR25 questionnaires between baseline and one year was around $70 \%$. There were no significant changes among the items of the QLQ-C30, in particular to physical or cognitive functioning (supplementary materials). Dyspnea and role functioning were the only symptoms to worsen to a not clinically relevant level but statistically significant degree between baseline and M6 $(p=0.0260$ and 0.0468 respectively), but disappeared at one year. There were no significant differences for urinary, bowel- 
related symptoms $(\mathrm{p}=1.0000$ and $\mathrm{p}=0.5726$ respectively) and sexual activity $(\mathrm{p}=0.1152)$ for the QLQ-PR25 scores at one year (fig. 3). At six months, a statistically - and clinically - significant worsening in sexual activity was observed $(p=0.0020$, medium value of +16.6 points $)$ as well as for expected androgen blockade-related symptoms $(\mathrm{p}=0.0080$, medium value of -5.6 points $)$.

\section{Discussion}

Global tolerance of the BLINDED protocol was satisfactory, in line with retrospective data for high-dose salvage WPRT in the literature [12]-[14], even in patients with a past history of prostatic irradiation.

Based on the measurements of the EORTC questionnaires, patient quality of life did not significantly worsen between baseline and one year. Sexual activity significantly decreased at six months, due to the androgen blockade-related castration. The increase in dyspnea at six months for $25 \%$ of the patients - although not clinically relevant and not present later - may also be attributed to androgen blockade as previously described in the literature [11].

Fifty-two per cent of patients had previous prostate bed or prostate exclusive radiotherapy. Bladder, sigmoid colon and small bowel ran the risk of being partially reirradiated. There were no increased urinary or digestive toxicity in these patients compared to those who had not previously been irradiated. These results are coherent with those from the other studies that considered pelvic reirradiation using stereotactic body radiotherapy [15]-[17] or even within the context of salvage WPRT directed by FCH-PET imaging [8]. Further study of the repair mechanisms in radiation injury to the pelvic tissues, and hence the feasibility of reirradiation, is highly recommended.

We should emphasize that the toxicity reported in this paper is based on an evaluation period of one 
year. Further evaluation after a longer follow-up period is required [18]. The limited number of patients also constitutes a weakness.

\section{Conclusions}

Rates of acute and one year urinary and digestive toxicity following whole-pelvis salvage irradiation with boost to oligometastatic FCH-PET-positive lymph nodes of prostate adenocarcinoma are acceptable. The moderate and transitory worsening of hypertension and sexual activity—but not digestive or bladder-related function—may be attributed to the combined androgen deprivation treatment. Later toxicity rates will be reported together with the treatment efficiency. The phase III BLINDED trial, which will compare these pelvic salvage strategies to long-term androgen blockade, will provide further data on the toxicity of these treatments.

\section{Acknowledgements}

This study was funded by Astellas.

\section{Bibliography}

[1] A. Lépinoy et al., "Pattern of occult nodal relapse diagnosed with (18)F-fluoro-choline PET/CT in prostate cancer patients with biochemical failure after prostate-only radiotherapy," Radiother. Oncol., vol. 111, no. 1, pp. 120-125, Apr. 2014.

[2] A. Wahart et al., "Intensity-modulated salvage radiotherapy with simultaneous integrated boost for local recurrence of prostate carcinoma: a pilot study on the place of PET-choline for guiding target volume delineation," Br. J. Radiol., vol. 89, no. 1058, p. 20150579, 2016.

[3] W. P. Parker et al., "Patterns of Recurrence After Postprostatectomy Fossa Radiation Therapy Identified by C-11 Choline Positron Emission Tomography/Computed Tomography," Int. J. Radiat. Oncol. Biol. Phys, vol. 97, no. 3, pp. 526-535, March 2017.

[4] J. J. Tosoian, M. A. Gorin, A. E. Ross, K. J. Pienta, P. T. Tran, and E. M. Schaeffer, "Oligometastatic prostate cancer: definitions, clinical outcomes, and treatment considerations," Nat. Rev. Urol., vol. 14, no. 1, pp. 15-25, Jan. 2017.

[5] P. Ost et al., "Surveillance or Metastasis-Directed Therapy for Oligometastatic Prostate Cancer Recurrence: A Prospective, Randomized, Multicenter Phase II Trial," J. Clin. Oncol., vol. 36, no. 5, pp. 446-453, Feb. 2018.

[6] U. Schick et al., "Androgen deprivation and high-dose radiotherapy for oligometastatic prostate cancer patients with less than five regional and/or distant metastases," Acta Oncol., vol. 52, no. 8, pp. 1622-1628, Nov. 2013. 
[7] M. Picchio et al., "(11)C-Choline PET/CT as a guide to radiation treatment planning of lymphnode relapses in prostate cancer patients," Eur. J. Nucl. Med. Mol. Imaging, vol. 41, no. 7, pp. 1270-1279, Jul. 2014.

[8] A. Fodor et al., "Toxicity and efficacy of salvage carbon 11-choline positron emission tomography/computed tomography-guided radiation therapy in patients with lymph node recurrence of prostate cancer," BJU Int., vol. 119, no. 3, pp. 406-413, 2017.

[9] G. Créhange et al., "Salvage reirradiation for locoregional failure after radiation therapy for prostate cancer: who, when, where and how?," Cancer Radiother., vol. 18, no. 5-6, pp. 524534, Oct. 2014.

[10] BLINDED.

[11] A. Goineau et al., "Prospective evaluation of quality of life 54 months after high-dose intensity-modulated radiotherapy for localized prostate cancer," Radiat. Oncol., vol. 8, p. 53, Mar. 2013.

[12] A. Bayley et al., "Clinical Application of High-Dose, Image-Guided Intensity-Modulated Radiotherapy in High-Risk Prostate Cancer," Int. J. Radiat. Oncol. Biol. Phys, vol. 77, no. 2, pp. 477-483, Jun. 2010.

[13] J. B. Adkison et al., "Phase I Trial of Pelvic Nodal Dose Escalation With Hypofractionated IMRT for High-Risk Prostate Cancer," Int. J. Radiat. Oncol. Biol. Phys., vol. 82, no. 1, pp. 184-190, Jan. 2012.

[14] M. Reis Ferreira et al., "Phase 1/2 Dose-Escalation Study of the Use of Intensity Modulated Radiation Therapy to Treat the Prostate and Pelvic Nodes in Patients With Prostate Cancer," Int. J. Radiat. Oncol. Biol. Phys., vol. 99, no. 5, pp. 1234-1242, Dec. 2017.

[15] B. A. Jereczek-Fossa et al., "Robotic image-guided stereotactic radiotherapy, for isolated recurrent primary, lymph node or metastatic prostate cancer," Int. J. Radiat. Oncol. Biol. Phys., vol. 82, no. 2, pp. 889-897, Feb. 2012.

[16] K. Decaestecker et al., "Repeated stereotactic body radiotherapy for oligometastatic prostate cancer recurrence," Radiat. Oncol., vol. 9, p. 135, Jun. 2014.

[17] T. Zilli, E. Benz, G. Dipasquale, M. Rouzaud, and R. Miralbell, "Reirradiation of Prostate Cancer Local Failures After Previous Curative Radiation Therapy: Long-Term Outcome and Tolerance," Int. J. Radiat. Oncol. Biol. Phys., vol. 96, no. 2, pp. 318-322, Jan. 2016.

[18] A. Sujenthiran et al., "National Population-Based Study Comparing Treatment-Related Toxicity in Men Who Received Intensity Modulated Versus 3-Dimensional Conformal Radical Radiation Therapy for Prostate Cancer," Int. J. Radiat. Oncol. Biol. Phys., vol. 99, no. 5, pp. 1253-1260, Jan. 2017. 


\section{Figures :}

Fig. 1 : (color online) Top panel: Schematic view of the patient population and treatment planning options. Prior RT for Group C : prior radiotherapy of the prostatic bed. Prior RT for Group D : prior prostate radiotherapy (external beam or brachytherapy). Bottom panel: Example of the treatment planning for one patient of Group B with FCH-PETpositive node into the right external iliac vessels and one left-posterior local relapse into the prostatic bed. Delineations of whole pelvic lymph nodes, bladder and rectum walls are shown.

Fig. 2 : Trial flow chart. $\mathrm{RP}=$ radical prostatectomy; $\mathrm{PB}=$ prostatic bed; $(\mathrm{EB}) \mathrm{RT}=(\mathrm{ex}-$ ternal beam) radiotherapy; BT = brachytherapy

Fig. 3 : (color online) Panel A : Number of patients with gastrointestinal (left) and genitourinary (right) CTCAE v4.0 toxic events at M1 ( $\leq 1$ month after the end of radiotherapy) and one year after the end of radiotherapy. The number of patients with urinary and bowel troubles at baseline are given for comparison. Patients of Group A (28/67) and Group B (4/67) did not receive prior radiotherapy; patients of Group C (29/67) and Group D (6/67) respectively received prior prostatic bed and prostate-exclusive radiotherapy.

Panel B : QLQ-PR25 score differences with time. 47/67 patients (70\%) completed all assessments with time. Incontinence aid only concerned a minority of patients and is not 


\section{ACCEPTED MANUSCRIPT}

shown. Sexual functioning only concerned a minority of the patients with sexual activity and is not shown.

Min = minimal difference, Max $=$ maximal difference, $\mathrm{Q} 1=$ first quartile, $\mathrm{Q} 3=$ third quartile. 
Early toxicity of the BLINDED

\section{Tables}

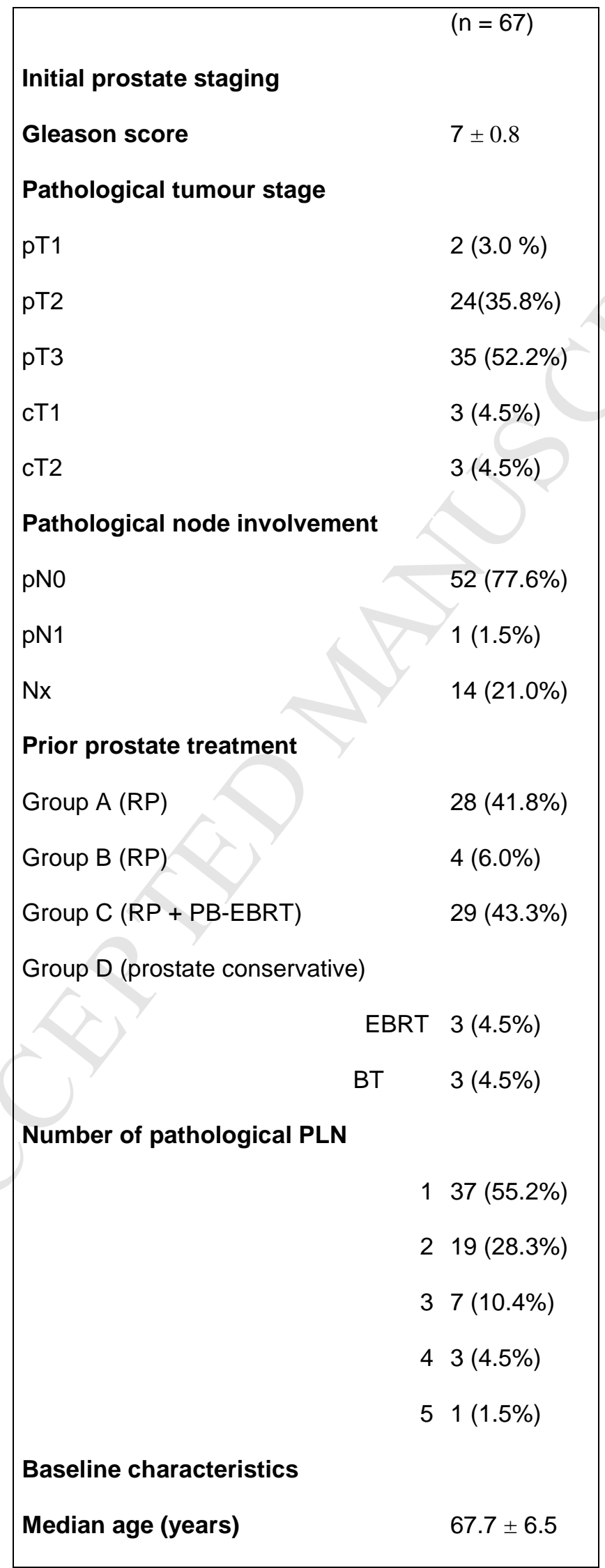


ECOG Performance Status

$062(92.5 \%)$

$15(7.5 \%)$

Hypertension

yes $32(47.8 \%)$

unknown $1(1.5 \%)$

Tobacco

yes $6(9.0 \%)$

unknown 13 (19.4\%)

Diabetes

yes $11(16.4 \%)$

unknown $1(1.5 \%)$

Digestive comorbidities

yes $7(10.4 \%)$

unknown $1(1.5 \%)$

Prior abdominal surgery

yes $15(22.4 \%)$

unknown $1(1.5 \%)$

Table 1 : Initial prostatic adenocarcinoma staging (TNM 2005) and baseline characteristics of the patients.

RP (radical prostatectomy); PB (prostatic bed); EBRT (external-beam radiotherapy); BT (brachytherapy); PLN (pelvic lymph node). Digestive comorbidities : gastric ulcer, gastroesophageal reflux, colonic polyps. Abdominal surgery : appendice, gall bladder, haemorrhoids, sigmoid colon. Radical prostatectomy was not counted. Quantitative variables : mean \pm standard deviation. Qualitative variables : number of subjects (\%). 


$$
(n=67)
$$

\begin{tabular}{|c|c|c|c|c|c|c|c|c|c|}
\hline Gastrointestinal & Grade & 1 & 2 & 3 & Genitourinary & Grade & 1 & 2 & 3 \\
\hline \multirow[t]{3}{*}{ Inconvenience } & Baseline & $1(1.5 \%)$ & - & - & Urgencies & Baseline & 7 (10.4\%) & $1(1.5 \%)$ & - \\
\hline & M1 & 21 (31.3\%) & $2(3.0 \%)$ & - & & M1 & $33(49.2 \%)$ & $8(11.9 \%)$ & - \\
\hline & 1-year & 11 (16.4\%) & - & - & & 1-year & 22 (32.8\%) & $2(3.0 \%)$ & \\
\hline \multirow[t]{3}{*}{ Diarrhea } & Baseline & $2(3.0 \%)$ & - & - & Incontinence & Baseline & 7 (10.4\%) & - & - \\
\hline & M1 & 37 (55.2\%) & 8 (11.9\%) & - & & M1 & 13 (19.4\%) & $2(3.0 \%)$ & - \\
\hline & 1-year & 10 (14.9\%) & $1(1.5 \%)$ & - & & 1-year & 17 (25.3\%) & - & $3(4.4 \%)$ \\
\hline \multirow[t]{3}{*}{ Bleeding } & Baseline & - & - & - & Hematuria & Baseline & - & - & - \\
\hline & M1 & $4(5.9 \%)$ & $1(1.5 \%)$ & - & & M1 & $3(4.4 \%)$ & - & - \\
\hline & 1-year & $4(5.9 \%)$ & $2(3.0 \%)$ & - & & 1-year & $2(3.0 \%)$ & $2(3.0 \%)$ & $1(1.5 \%)$ \\
\hline \multirow[t]{3}{*}{ Proctitis } & Baseline & $1(1.5 \%)$ & - & - & Pain & Baseline & - & - & - \\
\hline & M1 & 12 (17.9\%) & $2(3.0 \%)$ & - & & M1 & $7(10.4 \%)$ & - & - \\
\hline & 1-year & $5(7.4 \%)$ & $1(1.5 \%)$ & - & & 1-year & - & - & - \\
\hline \multirow[t]{6}{*}{ Pts with tox. } & Baseline & $2(3.0 \%)$ & - & - & Dysuria & Baseline & - & - & - \\
\hline & M1 & 47 (70.1\%) & 10 (14.9\%) & & & M1 & $3(4.4 \%)$ & - & - \\
\hline & 1-year & 20 (29.8\%) & 4 (5.9\%) & & & 1-year & - & $1(1.5 \%)$ & - \\
\hline & & & & & Pts with tox. & Baseline & 12 (17.9\%) & $1(1.5 \%)$ & - \\
\hline & & & & & & M1 & 40 (59.7\%) & $9(13.4 \%)$ & . \\
\hline & & & & & & 1-year & 32 (47.7\%) & $4(5.9 \%)$ & $3(4.4 \%)$ \\
\hline Cardiovascular & Grade & & 2 & 3 & & & & & \\
\hline \multirow[t]{3}{*}{ Hypertension } & Baseline & $29(43.3 \%)$ & $19(28.3 \%)$ & 6 (8.9\%) & & & & & \\
\hline & M1 & $12(17.9 \%)$ & 31 (46.2\%) & 12 (17.9\%) & & & & & \\
\hline & 1-year & 19 (28.3\%) & 15 (22.4\%) & $1(1.5 \%)$ & & & & & \\
\hline
\end{tabular}

Table 2 : Baseline, M1 ( $\leq 1$ month after the end of radiotherapy) and one-year urinary, digestive and cardiovascular events. A patient may present several symptoms. Example : a patient may have a grade 1 diarrhea and grade 2 digestive bleeding at M1. He thus would be counted in grade 1 and 
grade 2 number of patients with digestive toxicity at M1 (Pts with tox.). No grade 4 were reported.

Digestive inconvenience : constipation, flatulences, bloating, pain. Proctitis : haemorrhoids, anal pain. 


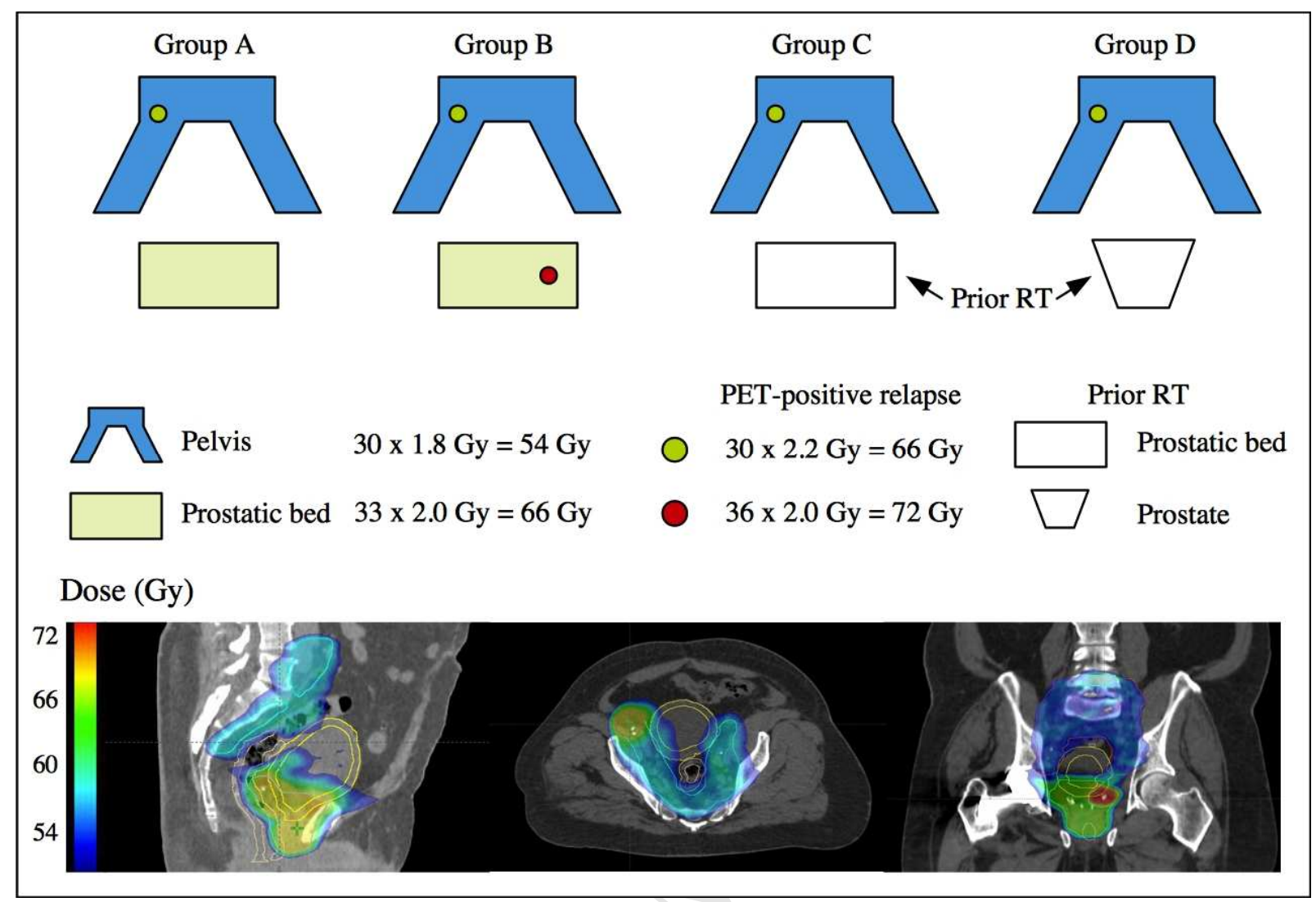




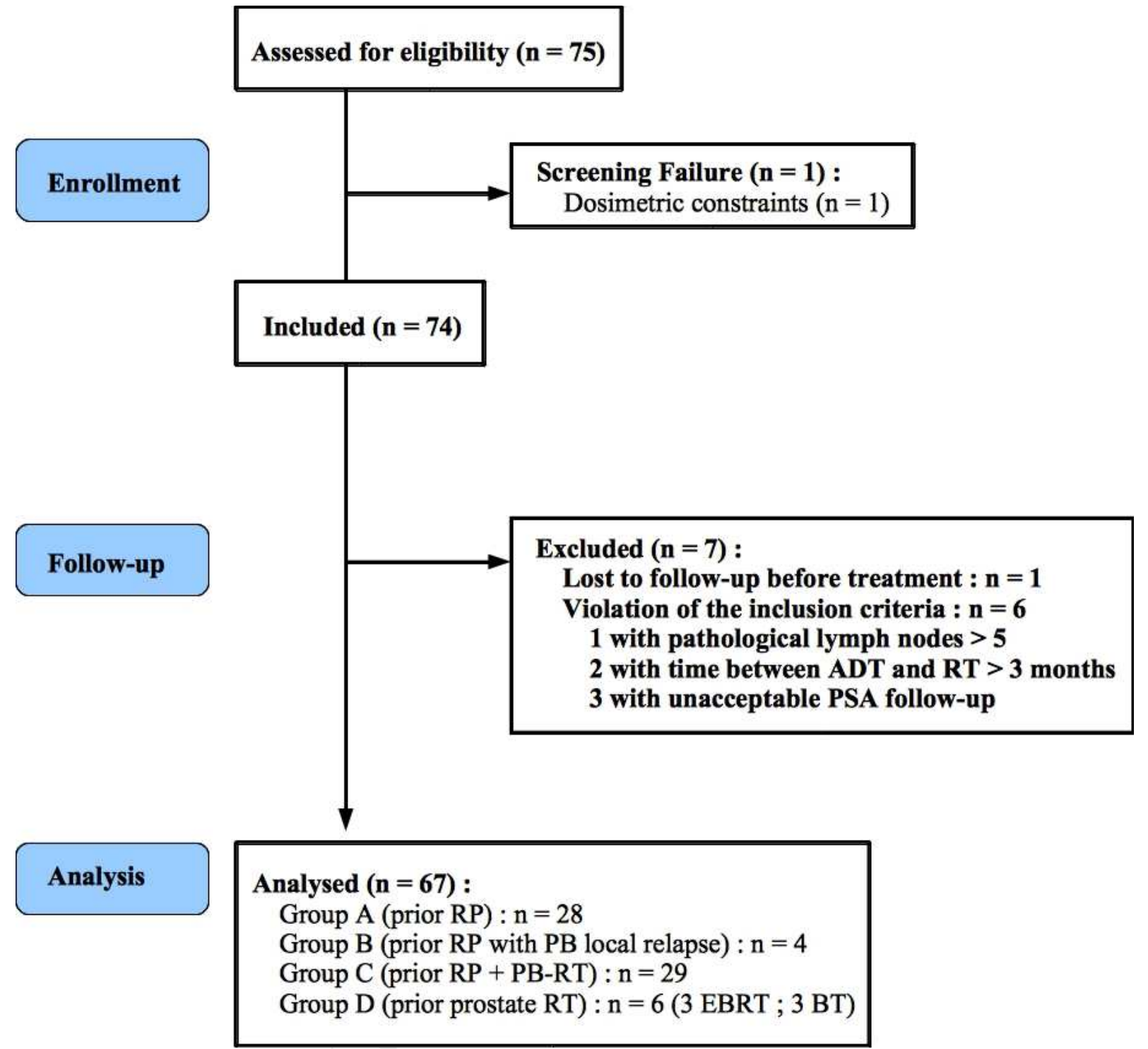


A.
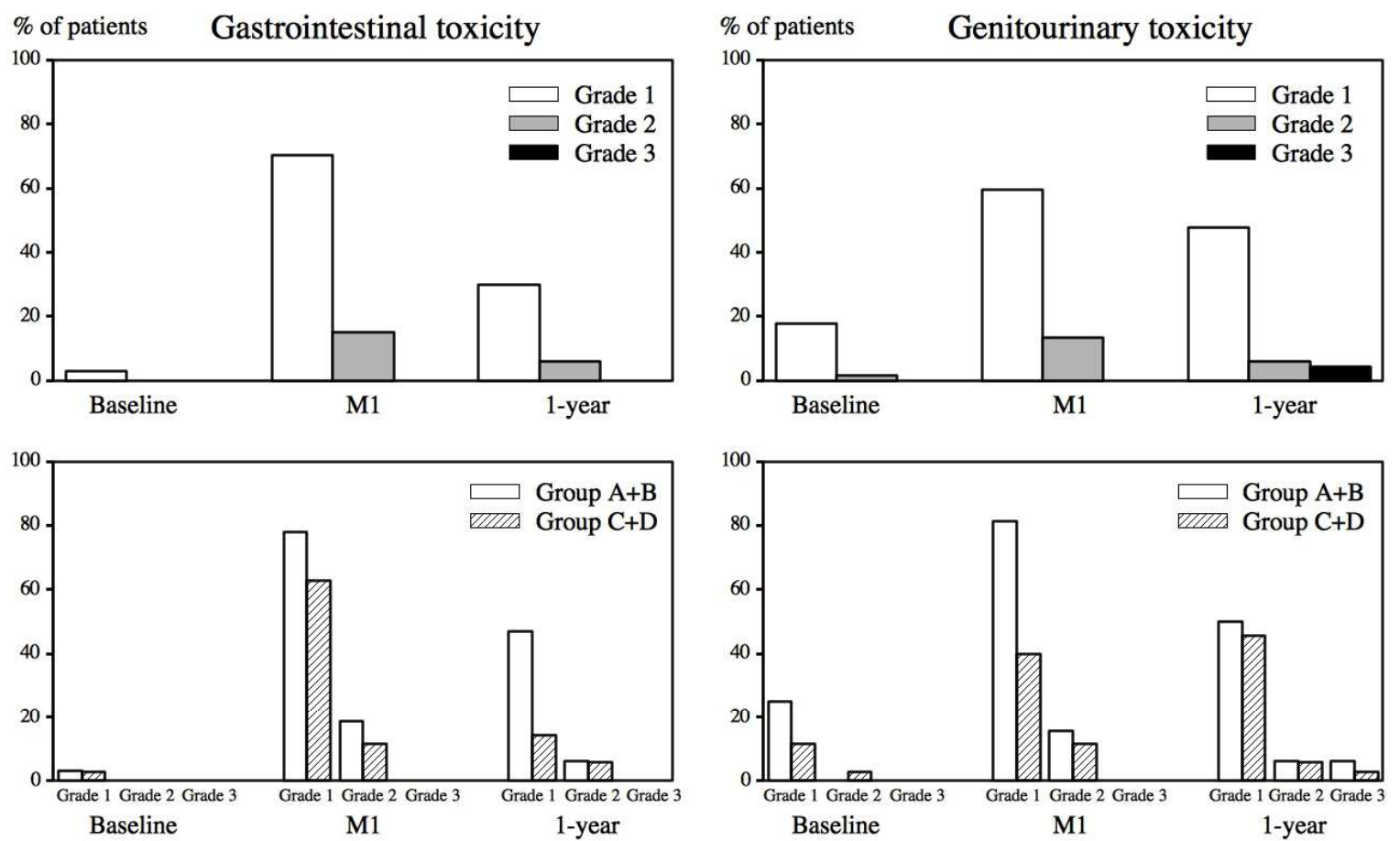

B. QLQ-PR25 Score Differences

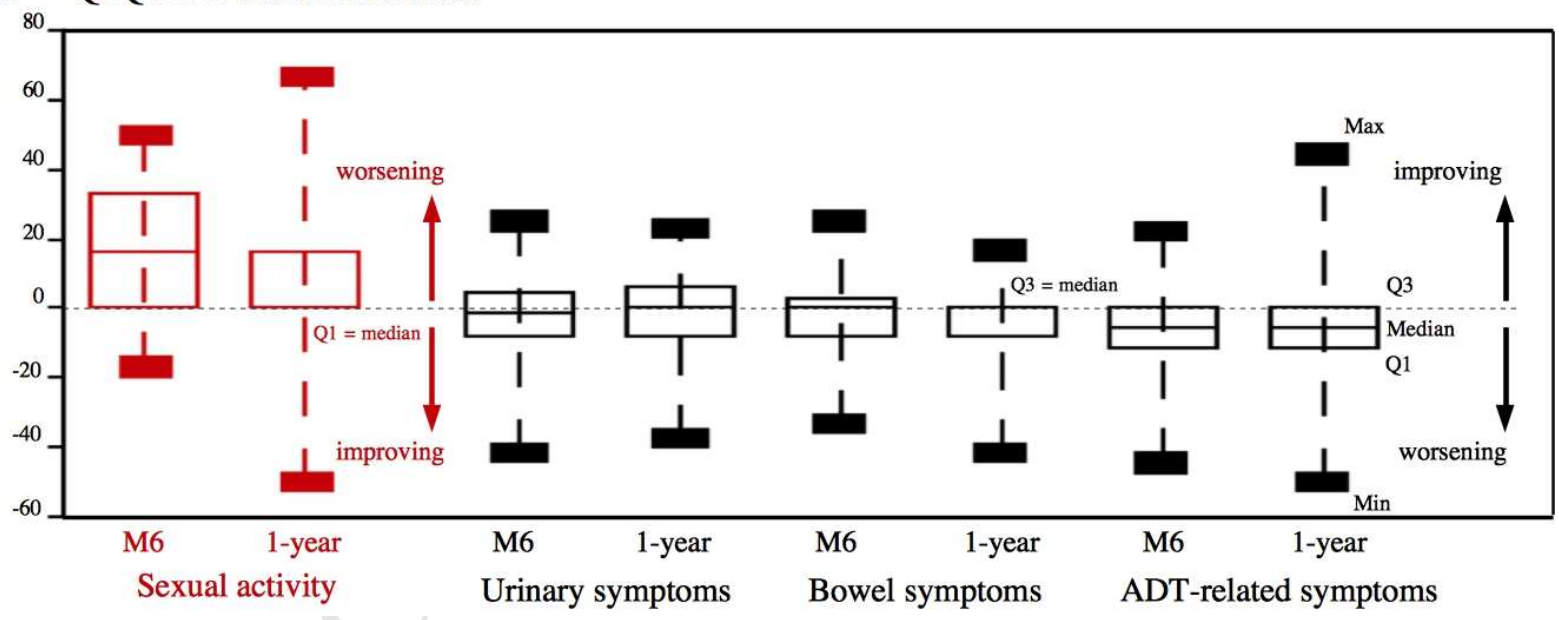




\section{Early toxicity of the BLINDED trial}

\section{Summary}

As the benefits of salvage pelvic radiotherapy in biochemically-recurrent prostate cancer following radical therapy is still unkown, the toxicity of such strategy matters.

BLINDED was a prospective multicenter phase II trial investigating a combination of six months androgen blockade with high-dose IG-IMRT salvage irradiation in pelvic oligometastatic patients detected by $18 \mathrm{~F}$-choline PET imaging.

Early toxicity until one year after radiotherapy was acceptable, in particular in patients with a past history of prostatic irradiation. 